\title{
Parental origin of mutations in sporadic cases of Treacher Collins syndrome
}

\author{
Alessandra Splendore ${ }^{1}$, Ethylin Wang Jabs ${ }^{2}$, Têmis Maria Félix ${ }^{3}$ and \\ Maria Rita Passos-Bueno*,1
}

${ }^{1}$ Centro de Estudos do Genoma Humano, Departamento de Biologia, Instituto de Biociências, Universidade de São Paulo, São Paulo, SP, Brazil; ${ }^{2}$ Department of Pediatrics, Medicine, and Surgery, Center for Medical Genetics, Johns Hopkins University School of Medicine, Baltimore, MD, USA; ${ }^{3}$ Departamento de Genética, Hospital de Clínicas de Porto Alegre, Universidade Federal do Rio Grande do Sul, Porto Alegre, RS, Brazil

In some autosomal dominant conditions, there is a correlation between new mutations and paternal age, with new mutations arising almost exclusively in the male germ line. To test this hypothesis in Treacher Collins syndrome, we analyzed 22 sporadic cases, determining the parental origin of the pathogenic mutation in $\mathbf{1 0}$ informative families. Mutations were found to be of both paternal and maternal origin, without a detectable parental age effect, confirming that a paternal age effect is not universal to all autosomal dominant disorders. A discussion on the parental origin of mutations and paternal age effect in other diseases is included.

European Journal of Human Genetics (2003) 11, 718-722. doi:10.1038/sj.ejhg.5201029

Keywords: Treacher Collins syndrome; TCOF1; parental age; sporadic mutation

\section{Introduction}

Treacher Collins syndrome (TCS; MIM 154500) is an autosomal dominant craniofacial disorder affecting the development of structures derived from the first and second branchial arches during early embryonic development. ${ }^{1}$ The resulting clinical features comprise downslanting palpebral fissures with lower eyelid coloboma, malar and maxilar hypoplasia, malformed ears and conductive hearing loss due to atresia of the external ear canal. $^{2}$ The gene underlying this condition, TCOF 1 , mapped to chromosome 5q32, was cloned in $1996 .{ }^{3}$ Pathogenic mutations in the TCOF1 gene are spread throughout its coding region, and are usually point mutations or small frameshift deletions and insertions, the majority of which are family-specific. Recent studies proposed the existence of mutational hot spots in TCOF1,

*Correspondence: Dr MR Passos-Bueno, Departamento de Genética, Instituto de Biociências, Universidade de São Paulo, Rua do Matão, 277, CEP 05508-900, Cidade Universitária, São Paulo, SP, Brazil. Tel: +55-113091-9910; Fax: +55-11-3091-7419; E-mail: passos@ib.usp.br Received 20 November 2002; revised 20 March 2003; accepted 1 April 2003 indicating that exons 23 and 24 are responsible for roughly one-third of all known pathogenic changes. ${ }^{4,5}$

The estimated prevalence of TCS is $1 / 50000$ live births, with $60 \%$ of the cases resulting from new mutations. ${ }^{6}$ It has been suggested by Jones et al., ${ }^{7}$ after reviewing 98 cases from the literature, that sporadic cases of TCS are associated with advanced paternal age. We are employing for the first time molecular methods to test if new mutations causing TCS arise preferentially in the germ line of older men. We investigated the parental origin of the pathogenic mutation in 22 sporadic cases of TCS and compared our results to the literature.

Subjects and methods

\section{Subjects}

We studied 70 TCS families (43 from Brazil, 22 from the USA, two from Argentina, one from Switzerland, one from Italy, and one from Finland) in which a pathogenic mutation had been previously identified. ${ }^{4,5}$ From this sample, 26 cases were familial, 42 were sporadic and two could not be classified. Parental origin of the pathogenic 
mutation was investigated in 22 sporadic cases for which there was available parental DNA.

DNA was extracted from whole blood according to standard techniques. ${ }^{8}$ PCR was performed with $40 \mathrm{ng}$ of DNA, using primers described elsewhere ${ }^{9-11}$ for TCOF1 exons and splice sites, or primers designed through Primer ${ }^{12}$ for intronic sequences. PCR products were analyzed through SSCP in native $5 \%$ acrylamide or $\mathrm{MDE}^{\mathrm{TM}}$ gels, as described elsewhere. ${ }^{4}$ Paternity was confirmed for all cases by means of five highly polymorphic tetranucleotide markers (D3S1754, D5S820, D6S477, D7S821, and D12S391) following standard procedures. Parental sex was confirmed through PCR amplification of X- and Y-chromosome specific markers (UniSTS:99017 and UniSTS:156591).

\section{Selection of informative markers}

A total of 17 single-nucleotide polymorphisms (SNPs) have already been described in the coding sequence of TCOF1, and three intronic alterations. ${ }^{4,10,11}$ The SNPs lying in the same or in adjacent exons to the one harboring the pathogenic mutations were ascertained to determine whether they were informative for determining the parental origin of the pathogenic mutation for each family. Intronic segments were screened through SSCP to detect novel SNPs. Introns up to 600 bp (IVS7, IVS8, IVS9, IVS11, IVS12, IVS14, IVS19, IVS20, and IVS24) were screened in full, whereas only portions ( $\sim 700 \mathrm{bp})$ adjacent to the exon of interest of larger introns were screened (IVS6 - distal, IVS13 - proximal and distal, IVS16 - distal, IVS17 proximal, IVS22 - distal, and IVS23 - proximal and distal fragments). Intronic primers were constructed via Primer3 and are available on request. Mobility shifts that indicated the sequence alteration would be informative were sequenced.

\section{Establishing the phase of the SNP alleles and the pathogenic mutation}

Two methods were used to establish the phase of the pathogenic mutation and the informative SNP: allelespecific (ARMS) amplification ${ }^{13}$ followed either by direct sequencing or analysis on denaturing 5\% acrylamide gel, and sequencing of cloned PCR products. Cloning of PCR products was performed with the TOPO $\mathrm{TA}^{\mathrm{TM}}$ Cloning Kit (Invitrogen, Carlsbad, CA, USA). Sequencing of cloned and genomic segments was performed in an ABIPrism Model 377 (Version 3.0), using the BigDye Terminator Cycle Sequencing kit (Applied Biosystems, Foster City, CA, USA).

\section{ARMS primer design and amplification}

Allele-specific primers were designed either aiming at amplifying different SNP alleles, or to distinguish between a mutant and the corresponding wild-type chromosome. ARMS primers were combined with the ones regularly used to amplify the exon containing the mutation, in the appropriate direction. The allele-specific primer sequences are available on request.

\section{Results}

Selection of informative families

An informative marker for determining the parental origin of a sporadic mutation must be present in a heterozygous state in the propositus and one parent, while the other is homozygous or, alternatively, when both parents are homozygous for different alleles. In five families, one of the SNPs already identified in TCOF1 satisfied these criteria: in families TCS10 and TCS18, the propositi were heterozygous for the c.1347 $\mathrm{T} \rightarrow \mathrm{C}$ SNP in exon 10; in family TCS24, the c.1611 G $\rightarrow$ A SNP in exon 11 was informative; and in families G1282 and TC512, the propositi were heterozygous for the c.3938 $\mathrm{C} \rightarrow \mathrm{T}$ SNP in exon 23 . For the 17 remaining families, we screened the intron(s) adjacent to the exon harboring the pathogenic mutation in order to characterize novel SNPs that could be used to distinguish between the maternal and paternal allele in the propositus.

\section{Identification of novel SNPs}

Three novel intronic SNPs were identified, two in intron 24 (IVS24+350 C $\rightarrow \mathrm{G}$ and IVS24+439 $\mathrm{C} \rightarrow \mathrm{A}$ ) and one in intron 6 (IVS6-701 $\mathrm{C} \rightarrow \mathrm{T}$ ). Three of the propositi with the pathogenic mutation in exon 24 were heterozygous for the IVS24+350 C/G polymorphism, whereas one additional patient was heterozygous for the IVS24+439 C/A alteration. The remaining patients with pathogenic mutations in exon 24 were homozygous for both SNPs. The IVS6-701 C/T SNP could be used to determine parental origin of the mutation for one family where the propositus had a mutation in exon 7. In total, 10 families were informative for determining the parental origin of the pathogenic mutation in the propositus, and for the remaining 12 no informative SNP could be found.

\section{Determining parental origin of mutation in informative families}

ARMS For the four informative families with the 5-bp deletion in exon 24, allele-specific primers were used to amplify each polymorphic allele separately. A denaturing gel analysis showed that in family TCS 15 the mutation was of paternal origin, whereas in families TCS 6, TCS 9 (Figure 1) and TCS 25, the mutation arose on the maternal chromosome. For propositi TCS24 and G1775, the allelespecific primers were constructed to discriminate between the deleted and wild-type alleles. Sequence analysis of the PCR-amplified products showed that in both cases the mutation was on the paternally inherited allele.

Cloning For those patients with the pathogenic mutation located in the same exon as the informative SNP (exons 10 
and 23), the PCR products were cloned and sequenced. Eight patients were analyzed in this manner, but only four were heterozygous for the polymorphism. Parental genotype for the polymorphism was established through SSCP analysis. In the four informative cases, the mutation was of paternal origin (Figure 2). Results for all informative families are presented in Table 1 .

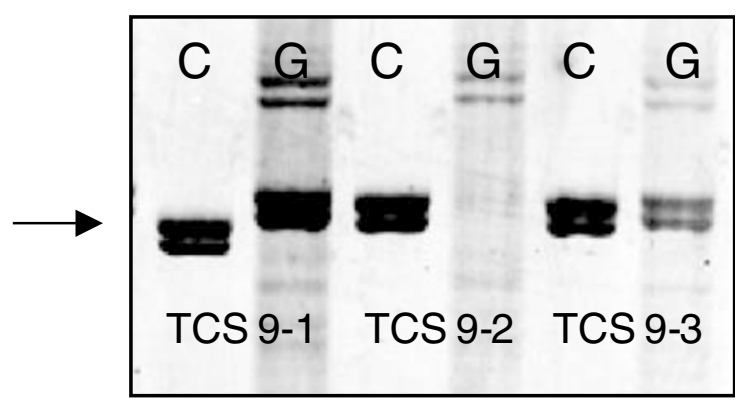

Figure 1 Denaturing gel electrophoresis of ARMS-amplified PCR products. The letter above each lane indicates the allele amplified for the IVS24+350 C/G SNP. The IVS24+350 $G$ primers also amplify an unspecific product. An arrow points to the allele showing a 5-bp deletion in the patient. Lanes 1, 2: patient TCS 9-1, C/G heterozygote; lanes 3, 4: patient's mother, a C/C homozygote, and lanes 5, 6: patient's father, also a C/G heterozygote.
Paternal and maternal age effect in TCOF1 mutations Mean paternal and maternal ages for all the studied cases are presented in Table 2. If we consider only those cases where the parental origin of the mutation could be established, we have a mean paternal age of 27.8 (seven cases) for those cases where the mutation was on the paternal chromosome, and a mean maternal age of 26.4 (three cases) for those of maternal origin.

\section{Discussion}

In the present study, we were able to determine the parental origin of a new mutation in 10 out of 22 families with sporadic TCS, and found that seven were of paternal origin and three were present in the maternally derived chromosome, with no preferential origin of new mutations in the male gametogenesis $(P=0.172)$. It is interesting to note, however, that in all three cases of maternal origin the mutation involved was the same, namely a 5-bp deletion in exon 24 (c4135-4139delGAAAA), that is the single frequent recurring mutation in TCS, found in roughly 15\% of all diagnosed cases. ${ }^{4,5,10}$ A greater number of cases should be investigated to see if maternal mutations are always restricted to this mutational hot spot or can also occur elsewhere in the gene.

Although the first classic genetic study by Jones et al. ${ }^{7}$ favored an increased paternal age for sporadic occurrences

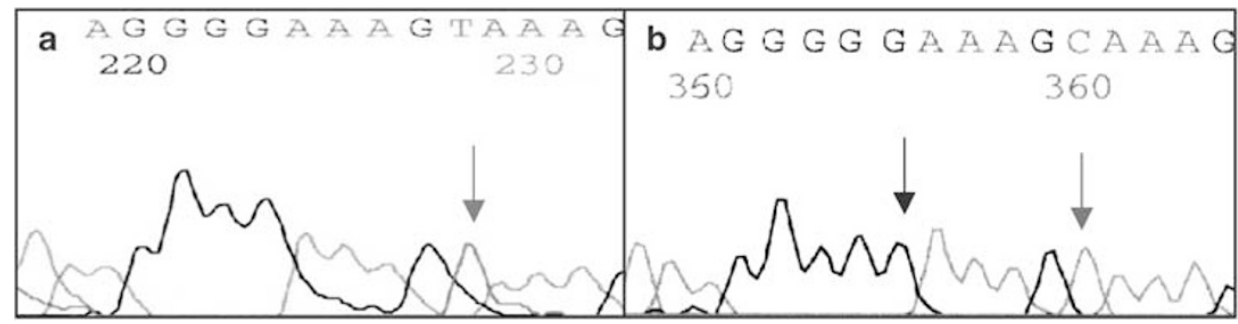

Figure 2 Sequencing of cloned PCR products. In this example (patient TC512), the wild-type sequence of exon 23 is in cis with the c.3938 T SNP (grey arrow) (a), and the allele with the c.3933^3934 G insertion (black arrow) in cis with the c.3938 C variant (grey arrow) (b).

Table 1 Informative families and parental origin of sporadic mutations

\begin{tabular}{|c|c|c|c|c|c|c|c|c|}
\hline Patient & Mutation & Exon & SNP & Method & $\begin{array}{c}\text { Parent of } \\
\text { origin }\end{array}$ & $\begin{array}{l}\text { Father's age } \\
\text { (years) }\end{array}$ & $\begin{array}{c}\text { Mother's age } \\
\text { (years) }\end{array}$ & Nationality \\
\hline G1775 & c.720-727delAGCACCCC & 7 & IVS6-710 C $\rightarrow T$ & ARMS & Father & 35.7 & 31.6 & American \\
\hline TCS 10 & c.1408-1409delAG & 10 & c. $1347 \mathrm{~T} \rightarrow \mathrm{C}$ & Cloning & Father & 25 & 28 & Brazilian \\
\hline TCS 18 & c.1406-1409delAGAG & 10 & c. $1347 \mathrm{~T} \rightarrow \mathrm{C}$ & Cloning & Father & 25 & 22 & Brazilian \\
\hline TCS 24 & c.2018-2025delCAGTCACC & 13 & c. $1611 \mathrm{G} \rightarrow \mathrm{A}$ & ARMS & Father & 20.2 & 17.9 & Brazilian \\
\hline G1282 & c.3639delG & 23 & c.3938 C $\rightarrow T$ & Cloning & Father & 34.3 & 33.4 & American \\
\hline TC512 & c. $3933^{\wedge} 3934$ insG & 23 & c. $3938 \mathrm{C} \rightarrow \mathrm{T}$ & Cloning & Father & 34.8 & 33.2 & American \\
\hline TCS 6 & c. $4135-4139$ delGAAAA & 24 & IVS24+350 C $\rightarrow \mathrm{G}$ & ARMS & Mother & 26.0 & 20.7 & Brazilian \\
\hline TCS 9 & c.4135-4139delGAAAA & 24 & IVS24+350 C $\rightarrow \mathrm{G}$ & ARMS & Mother & 26 & 34 & Brazilian \\
\hline TCS 15 & c. $4135-4139$ delGAAAA & 24 & IVS24+439 C $\rightarrow$ A & ARMS & Father & 20 & 17 & Brazilian \\
\hline TCS 25 & c.4135-4139delGAAAA & 24 & IVS24+350 C $\rightarrow \mathrm{G}$ & ARMS & Mother & 34.1 & 24.7 & Brazilian \\
\hline
\end{tabular}


of TCS, mean paternal age among parents of all sporadic TCS cases in our sample was $30.3 \pm 6.6$ years, with a median of 29 years. If we take into account only Brazilian families (13 cases), which represent the largest sample, mean paternal age falls to $26.8 \pm 4$.1. As there are no population means for paternal age for the Brazilian population available from vital statistics, a control group of 88 families with a cleft lip/palate proband from the same socioeconomic background as the Brazilian TCS families was ascertained, yielding a mean paternal age of $27.9 \pm 6.7$. There is no statistically significant difference between the mean paternal age in the control group when compared to the fathers of TCS probands from Brazil $(P=0.5266)$ or the total sample $(P=0.1555)$. Even considering only those cases where the new mutation in TCOF1 was shown to be of paternal origin, paternal age (27.8) is not increased when compared to the mean paternal age for the Brazilian population.

The list of diseases in which the parental origin of new mutations was elucidated through the use of molecular analysis is listed in Table 3. Exclusive paternal origin of new mutations with a paternal age effect was found for mutations in the FGFR2 (Apert, Crouzon and Pfeiffer syndromes), ${ }^{14,15}$ FGFR3 (achondroplasia), ${ }^{16}$ and RET (MEN 2A and MEN 2B) ${ }^{17,18}$ genes. Until recently, the most favored explanation for these observations was that the mutations arise during cell replication, and the predominance of new mutations in the male germ line is due to the

Table 2 Mean parental age for tested families

\begin{tabular}{lccr}
\hline & Mean paternal age & Median & Cases \\
\hline Total & $30.3 \pm 6.6$ & 29 & 21 \\
Paternal origin only & 27.8 & 25 & 7 \\
Total & $27.4 \pm 6.4$ & 27.1 & 22 \\
Maternal origin only & 26.4 & 24.7 & 3 \\
\hline
\end{tabular}

increased number of germ line cell divisions in spermatogenesis compared with that in oogenesis. ${ }^{19,20}$ As spermatogonia continue to replicate throughout a man's life, the sperm of older men would accumulate a greater number of mutations, thus producing the observed paternal age effect. But if the number of cell replications is the only factor associated with new mutations, an increase of paternal age would have been observed in all syndromes that show a preferential paternal origin of mutations, and this correlation is not observed in various conditions, including the present report.

Some authors suggested that the preferential origin of paternal mutations is correlated to the type of mutation, implying that base substitutions occur primarily during the male gametogenesis, while other types of mutations, such as deletions and insertions, are more likely to arise during the female gametogenesis. ${ }^{21,22}$ This difference would result from intrinsic peculiarities in the male and female germinative cells, such as different methylation patterns or in the efficiency of the mechanism of repair. However, the correlation between single-nucleotide transitions and transversions and paternal origin of mutations failed to be confirmed when parental origins of mutations were determined for other conditions (Table 3 and references therein).

A recent work ${ }^{23}$ tested the relation between paternal age and sperm mutation frequency for the FGFR3 c.1138G $\rightarrow \mathrm{A}$ mutation that causes achondroplasia. Their data indicate that the paternal age effect found in achondroplasia cannot be explained by the increased number of mutations in the sperm of older men. The authors suggest that the mutation may confer a selective advantage either to the sperm or the zygote carrying it. A similar selective advantage to sperm carrying FGFR mutations had already been suggested by Oldrigde et al. ${ }^{24}$ It is therefore possible that the selection of sperm or zygotes carrying the pathogenic change is dependent on the function of the mutated genes, but it is not yet clear how this would cause an increase of affected children in the offspring of older men.

Table 3 Parental origin of mutation in other syndromes

\begin{tabular}{|c|c|c|c|c|c|c|}
\hline Syndrome & Gene & Type of mutation & $\begin{array}{l}\text { Paternal } \\
\text { origin (\%) }\end{array}$ & $\begin{array}{l}\text { Informative } \\
\text { families }\end{array}$ & $\begin{array}{c}\text { Increased } \\
\text { paternal age }\end{array}$ & References \\
\hline Achondroplasia & FGFR3 & Missense & 100 & 40 & Yes (35.86) & 16 \\
\hline Apert & FGFR2 & Missense & 100 & 57 & Yes (33.3) & 14 \\
\hline Crouzon & FGFR2 & Missense, splice (all base changes) & 100 & 11 & Yes (34.8) & 15 \\
\hline Pffeifer & FGFR2 & Missense, splice (all base changes) & 100 & 11 & Yes (33.65) & 15 \\
\hline MEN2A & RET & Missense & 100 & 10 & Yes (39.3) & 17 \\
\hline MEN2B & $R E T$ & Missense & 96.1 & 26 & Yes (33) & 18,25 \\
\hline Neurofibromatosis & NF1 & Indirect testing & 91.6 & 32 & No $(30.02)$ & 26,27 \\
\hline Retinoblastoma & $R B$ & Indirect testing & 82 & 49 & No $(29.7)$ & 28 \\
\hline Rett & MECP2 & Missense, nonsense, splice, insertion, deletion & 96 & 27 & No (31.3) & 29 \\
\hline Tuberous sclerosis & TSC2 & Missense, nonsense, splice, insertion, deletion & 41.7 & 12 & No $(29.6)$ & 30 \\
\hline Treacher Collins & TCOF1 & Nonsense, splice, insertion, deletion & 70 & 10 & No (30.3) & Present study \\
\hline
\end{tabular}


In conclusion, we show that new mutations in TCOF1 are not exclusivelly paternal and show no paternal age effect. An investigation of parental origin of mutations in a larger sample will be helpful to determine if there is a predominance of mutations arising on the male gametogenesis and if maternal mutations occur only at the exon 24 hot spot or can also appear elsewhere in the gene.

\section{Acknowledgements}

The authors thank the TCS families for their collaboration to this study and Drs Sérgio Matioli and Rivka Glaser for valuable help. The financial support of FAPESP, CNPq and Pronex is also gratefully acknowledged. MRPB is supported in part by an International Research Scholars grant from the Howard Hughes Medical Institute. This work was supported in part by NIH P6O DE13078, NIH HD24061 and NIH RROOO52 (EWJ).

\section{References}

1 Poswillo D: The pathogenesis of the Treacher Collins syndrome (mandibulofacial dysostosis). Br J Oral Surg 1975; 13: 1-26.

2 Franceschetti A, Klein D: Mandibulo-facial dysostosis, new hereditary syndrome. Acta Ophthal 1949; 27: 143-224.

3 The Treacher Collins Syndrome Collaborative Group: Positional cloning of a gene involved in the pathogenesis of Treacher Collins syndrome. Nat Genet 1996; 12: 130-136.

4 Splendore A, Silva EO, Alonso LG et al: High mutation detection rate in TCOF1 among Treacher Collins syndrome patients reveals clustering of mutations an 16 novel pathogenic changes. Hum Mutat 2000; 16: 315-322.

5 Splendore A, Jabs EW, Passos-Bueno MR: Screening of TCOF1 in patients from different populations, confirmation of mutational hot spots and identification of a novel missense mutation that suggests an important functional domain in the protein treacle. J Med Genet 2002; 39: 493-495.

6 Jones KL: Smith's recognizable patterns of human malformation, 5th edn., Philadelphia: Saunders, 1997.

7 Jones KL, Smith DW, Harvey MA, Hall BD, Quan L: Older paternal age and fresh gene mutation, date on additional disorders. J Pediatr 1975; 86: 84-88.

8 Miller SA, Dykes DD, Polesky HF: A simple salting out procedure for extracting DNA from human nucleated cells. Nucleic Acids Res 1988; 16: 1215

9 Gladwin AJ, Dixon J, Loftus SK et al: Treacher Collins syndrome may result from insertions, deletions or splicing mutations, which introduce a termination codon into the gene. Hum Mol Genet 1996; 5: 1533-1538.

10 Edwards SJ, Gladwin AJ, Dixon MJ: The mutational spectrum in Treacher Collins syndrome reveals a predominance of mutations that create a premature-termination codon. Am J Hum Genet 1997; 60: 515-524.

11 Wise CA, Chiang LC, Paznekas WA et al: TCOF1 gene encodes a putative nucleolar phosphoprotein that exhibits mutations in Treacher Collins Syndrome throughout its coding region. Proc Natl Acad Sci USA 1997; 94: 3110-3115.
12 WWW Primer Picking (Primer3): The Whitehead Institute for Biomedical Research. Cambridge, MA. http://www-genome. wi.mit.edu/cgi-bin/primer/primer3.cgi.

13 Newton CR, Graham A, Heptinstall LE et al: Analysis of any point mutation in DNA. The amplification refractory mutation system (ARMS). Nucleic Acids Res 1989; 17: 2503-2516.

14 Moloney DM, Slaney SF, Oldridge M et al: Exclusive paternal origin of new mutations in Apert syndrome. Nat Genet 1996; 13: 48-53.

15 Glaser RL, Jiang W, Boyadjiev SA et al: Paternal origin of FGFR2 mutations in sporadic cases of Crouzon syndrome and Pfeiffer syndrome. Am J Hum Genet 2000; 66: 768-777.

16 Wilkin DJ, Szabo JK, Cameron R et al: Mutations in fibroblast growth-factor receptor 3 in sporadic cases of achondroplasia occur exclusively on the paternally derived chromosome. Am $J$ Hum Genet 1998; 63: 711-716.

17 Schuffenecker I, Ginet N, Goldgar D et al and Le Groupe d'Étude des Tumeurs à Calcitonine: Prevalence and parental origin of de novo RET mutations in multiple endocrine neoplasia type $2 \mathrm{~A}$ and familial medullary thyroid carcinoma. Am J Hum Genet 1997; 60: 233-237.

18 Carlson KM, Bracamontes J, Jackson CE et al: Parent-of-origin effects in multiple endocrine neoplasia type 2B. Am J Hum Genet 1994; 55: 1076-1082.

19 Vogel F, Rathenberg R: Spontaneous mutation in man. Adv Hum Genet 1975; 5: 223-318.

20 Drost JB, Lee WR: Biological basis of germline mutation: comparisons of spontaneous germline mutation rates among drosophila, mouse, and human. Environ Mol Mutagen 1995; 26 (Suppl): 48-64.

21 Ketterling RP, Vielhaber E, Bottema CDK et al: Germ-line origins of mutation in families with hemophilia $\mathrm{B}$ : the sex ratio varies with the type of mutation. Am J Hum Genet 1993; 52: 152-166.

22 Crow JF: The origins, patterns and implications of human spontaneous mutation. Nat Rev Genet 2000; 1: 40-47.

23 Tiemann-Boege I, Navidi W, Grewal R et al: The observed human sperm mutation frequency cannot explain the achondroplasia paternal age effect. Proc Natl Acad Sci USA 2002; 99: 14952-14957.

24 Oldridge M, Lunt PW, Zackai EH et al. Genotype-phenotype correlation for nucleotide substitutions in the IgII-IgIII linker of FGFR2. Hum Mol Genet 1997; 6: 137-143.

25 Kitamura Y, Scavarda N, Wells Jr AS, Jackson CE, Goodfellow PJ: Two maternally derived missense mutations in the tyrosine kinase domain of the RET protooncogene in a patient with de novo MEN 2B. Hum Mol Genet 1995; 4: 1987-1988.

26 Jadayel D, Fain P, Upadhyaya $\mathrm{M}$ et al: Paternal origin of new mutations in Von Recklinghausen neurofibromatosis. Nature 1990; 343: 558-559.

27 Lázaro C, Gaona A, Ainsworth $\mathrm{P}$ et al: Sex differences in mutational rate and mutational mechanism in the NF1 gene in neurofibromatosis type 1 patients. Hum Genet 1996; 98: 696-699.

28 Dryja TP, Morrow JF, Rapaport JM: Quantification of the paternal allele bias for new germline mutations in the retinoblastoma gene. Hum Genet 1997; 100: 446-449.

29 Trappe R, Laccone F, Cobilanschi J et al: MECP2 mutations in sporadic cases of Rett syndrome are almost exclusively of paternal origin. Am J Hum Genet 2000; 68: 1093-1101.

30 Roberts PS, Chung J, Jozwiak S et al: SNP identification, haplotype analysis, and parental origin of mutations in TSC2. Hum Genet 2002; 111: 96-101. 